\title{
Solar Eclipses Observed from Antarctica
}

\author{
Jay M. Pasachoff \\ Williams College - Hopkins Observatory and California Institute of Technology \\ email: eclipse@williams.edu
}

\begin{abstract}
Aspects of the solar corona are still best observed during totality of solar eclipses, and other high-resolution observations of coronal active regions can be observed with radio telescopes by differentiation of occultation observations, as we did with the Jansky Very Large Array for the annular solar eclipse of 2012 May 20 in the US. Totality crossing Antarctica included the eclipse of 2003 November 23, and will next occur on 2021 December 4; annularity crossing Antarctica included the eclipse of 2008 February 7, and will next occur on 2014 April 29. Partial phases as high as $87 \%$ coverage were visible and were imaged in Antarctica on 2011 November 25, and in addition to partial phases of the total and annular eclipses listed above, partial phases were visible in Antarctica on 2001 July 2011, 2002 December 4, 2004 April 19, 2006 September 22, 2007 September 11, and 2009 January 26, and will be visible on 2015 September 13, 2016 September 1, 2017 February 26, 2018 February 15, and 2020 December 14. On behalf of the Working Group on Solar Eclipses of the IAU, the poster showed the solar eclipses visible from Antarctica and this article shows a subset (see www.eclipses.info for the full set). A variety of investigations of the Sun and of the response of the terrestrial atmosphere and ionosphere to the abrupt solar cutoff can be carried out at the future eclipses, making the Antarctic observations scientifically useful.
\end{abstract}

Keywords. eclipse, atmosphere, ionosphere

Scientific observations for the total eclipses ordinarily include electronic imaging of the corona to compare with simultaneous space observations of the Sun. Our links to maps and other items of coordination can be found at http://www.eclipses.info, the site of the IAU Program Group on Public Education at the Times of Eclipses and of the IAU Working Group on Eclipses. Special filters must be used to reduce the solar disk to a safe intensity during the partial phases; only during totality can one look safely without filters.

Since the paths of annularity or totality cross only a narrow band on Earth, observatories and ground stations in the path can get useful scientific observations. For example, the solar occultation that my group observed with the Jansky Very Large Array in 2012 should improve the spatial resolution of solar active regions, bringing radio observations to a finer resolution than ground-based optical observations or space optical or ultraviolet observations. Observations of temperature, ionospheric response, and other parameters during eclipses can be interpreted to show the response of the terrestrial atmosphere and ionosphere to an abrupt cutoff of insolation. For the total solar eclipses that are uniquely available from Antarctica (next on 2021 December 4), a wide range of imaging and spectroscopy should be obtained to help follow the Sun over the solar-activity cycle. 


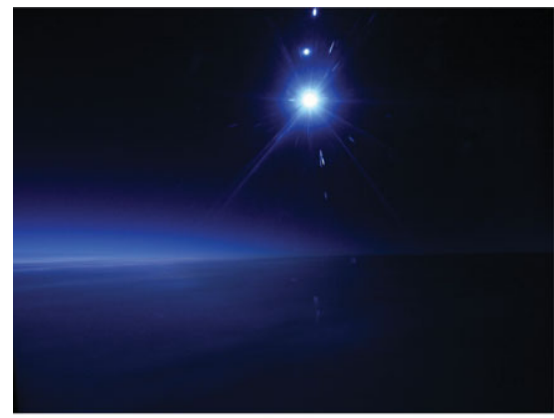

(a)

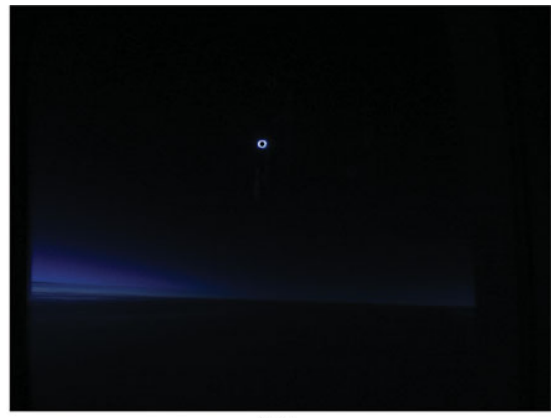

(b)

Figure 1. Wide-angle views out the windows of the Qantas flight to view the 2003 total solar eclipse over Antarctica, showing the umbral shadow approaching (left) and the solar corona (right) while the airplane was immersed in the shadow.

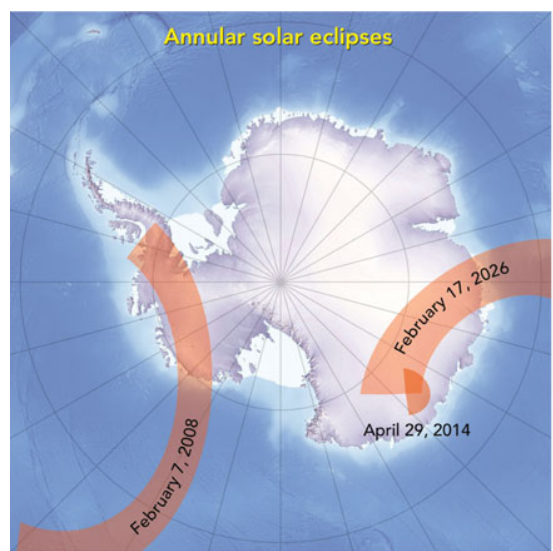

(a)

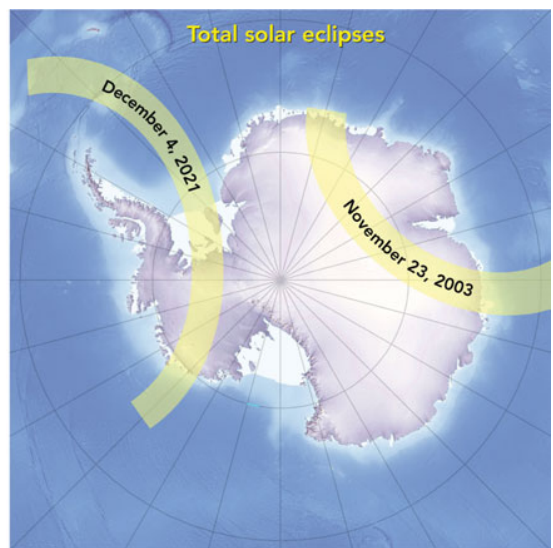

(b)

Figure 2. Notable past and future Antarctic (a) annular and (b) total solar eclipses.

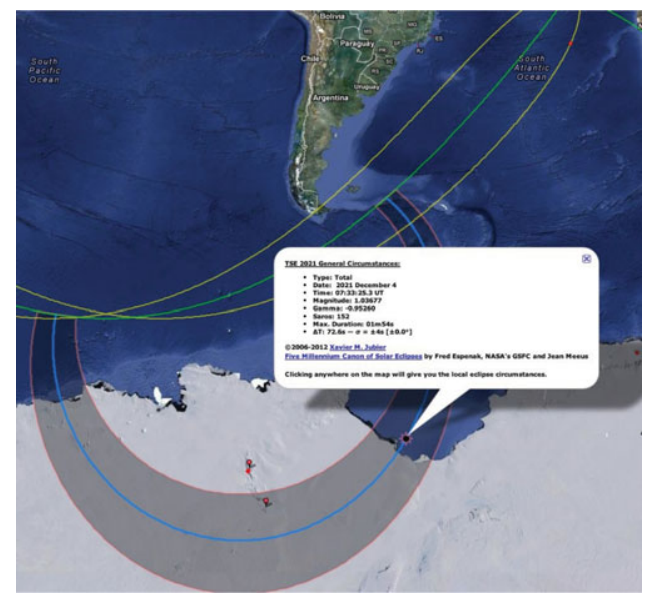

Figure 3. Circumstances of the 2021 December 4 total solar eclipse shown in Google Maps. Courtesy of Xavier Jubier. http://xjubier.free.fr/en/site_pages/solar_eclipses/TSE_2021_ GoogleMapFull.html 


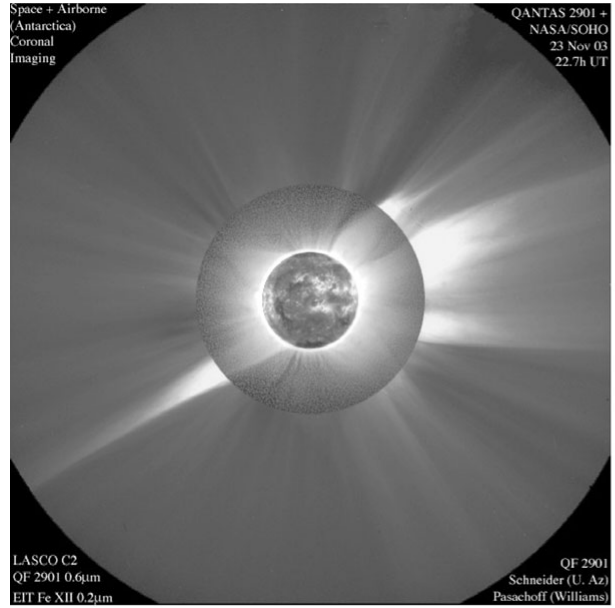

(a)

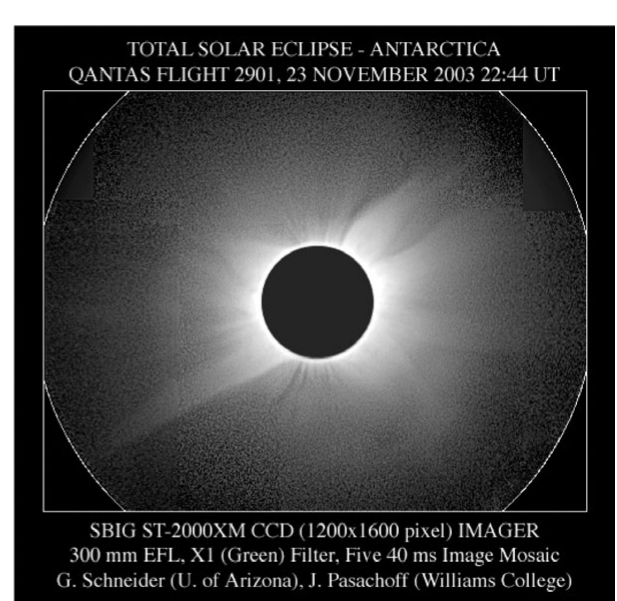

(b)

Figure 4. (a) An image composite of outer corona with the second (C2) telescope of the Large Angle Spectroscopic Coronagraph on the European Space Agency/National Aeronautics and Space Administration's Solar and Heliospheric Observatory (SOHO/LASCO C2), mid and inner corona from QF 2901 (airborne over Antarctica) by JMP and Glenn Schneider (U. Arizona), and the chromosphere with SOHO's Extreme-ultraviolet Imaging Telescope (EIT), all nearly contemporaneous. (b) On the right is a wide-field mosaic image of the 2003 total solar eclipse made from several different exposures made from QF 2901, to compensate for the wide dynamic range of the solar corona, which falls off in intensity by a factor of about 1,000 within about one solar radius.

With a total eclipse and an annular eclipse each occurring somewhere on Earth about every 18 months but less often than every 300 years at a given location, we should be using all telescopes and other scientific instruments in eclipse paths to their fullest possible extent.

\section{Acknowledgements}

JMP's research on the solar eclipses of 2012 is supported by grant AGS-1047726 from the Solar Physics Program of the Division of Atmospheric and Geospace Sciences of the US National Science Foundation. For the 2003 eclipse, Jay Pasachoff was part of the EurAstro Association team on the Croydon Travel chartered 747 over Antarctica. I thank Michael Zeiler (eclipse-maps.com) for the maps. See http://www.williams.edu/ astronomy/eclipse.

\section{References}

Pasachoff, Jay M. 2009, "Solar Eclipses as an Astrophysical Laboratory", Nature, June 11, 459, 789-795, DOI 10.1038/nature07987.

Pasachoff, Jay M. 2009, "Scientific Observations at Total Solar Eclipses", Research in Astronomy and Astrophysics, 9, 613-634. http://www.raa-journal.org/raa/index.php/raa/ article/view/182. 